\title{
Wetland flora of Betkot lake, far western Nepal
}

\section{Ramesh Basnet $^{1} \bowtie$, Dolraj Luintel ${ }^{1}$, Krishnaram Bhattarai $^{2}$, Mohandev Joshi ${ }^{3}$ and Kalyan Sapkota ${ }^{4}$}

\author{
${ }^{1}$ National Herbarium and Plant Laboratories, Lalitpur; ${ }^{2}$ Department of Plant Resources, \\ Kathmandu; ${ }^{3}$ Department of Environment, Kathmandu 4 Regional Forest Directorate, Surkhet \\ rameshwetlandnp@gmail.com
}

\begin{abstract}
The tropical wetland flora from western Nepal is less explored in comparison to the temperate and alpine region.This study was therefore undertaken to document the vascular plants found on the foot trail of surrounding BetkotLake, in Chure (Siwalik) hills of Kanchanpur in far western Nepal. The study documented a total of 63 plant species of 40 families belonging to 4 life forms: herbs (31 species), shrubs (14 species), trees (15 species) and climbers ( 3 species). This present study could be a baseline for further research.
\end{abstract}

Keywords: Betkotlake,flora documentation, wetland vegetation

\section{INTRODUCTION}

The Ramsar Convention in 1971 uses a broad definition of the types of wetlands covered in its mission, including swamps and marshes, lakes and rivers, wet grasslands and peatlands, oases, estuaries, deltas and tidal flats, near-shore marine areas, mangroves and coral reefs, and human-made sites such as fish ponds, rice paddies, reservoirs, and salt pans (http://wwf. panda.org/what_we_do/how_we_work/policy/conventions/ramsar/). Wetlands are regarded as the most productive and dynamic ecosystem of the earth and also called as biological supermarket, natural infrastructure, kidneys of the landscape, carbon dioxide sink and climate stabilizer. Nepal Biodiversity Strategy 2002 has recognized wetland as one of the sectoral strategies (others are forest, conserved area, rangeland, mountain and agriculture). The wetlands are in vulnerable status around the world. In fact, wetlands are not wasting land, but they are waiting land (Bhandariet al., 1994).

Nepal is rich in wetlands. It has 6,000 rivers and 5,358 lakes (http://www.nepallake.gov.np/). There are some studies about flora of wetlands in Nepal. Siwakoti (2006) recorded 720 species of vascular plants from the study of wetland flora of Terai regions. Sah (1997) summarized the biological resources of KoshiTappu Wildlife Reserve, eastern Terai. Likewise,Lamsalet al. (2014) documented 45 species of aquatic macrophytes and 54 species of terrestrial/riparian speciesfrom the study of Ghodaghodilake of Kailali district.In addition to Terai wetlands, wetland flora of Chitwan (Dangol, 2000-2001; Dangol et al., 2014) and Myagdi (Basnet et al., 2012) were also reported. The information about wetland flora of Nepal is not sufficient (Bhandari, 2008; Siwakoti, 2006). The tropical wetland flora from the Western part of Nepal is less explored in comparison to the temperate and alpine region. Hence, this study was conducted to document the plants of the Betkot lake area to provide baseline information useful for the 'Nepal Flora' publication. 


\section{MATERIALS AND METHODS}

\section{Study area}

The scenic Betkotlake has special value for maintaining genetic and ecological diversity that merit legal protection (MFSC, 2002). It is situated at Daijee-5, Kanchanpur district at the altitude $490 \mathrm{~m}$ asl, having water body size $4 \mathrm{ha}$. and $915 \mathrm{~m}$ perimeter including foot trail, representing the geologically and hydrologically fragile Chure/Siwalik region. The study route was the complete foot trail of the lake. It can be reached by Kathmandu-Dhangadi-Daijee-Betkot.

\section{Plant collections}

Plants samples (mainly phanerogams, however ferns and mushrooms) were collected during February 1-4 and June 21-24 of 2013 following standard plant collection methods. Plants were identified with the help of experts and standard literatures (Stainton, 1972; Bhandari, 1998; DPR, 2001, 2002; Siwakoti, 2006; Basnetet al., 2012; Fraser-Jenkins et al., 2015) and the deposited herbarium in the National Herbarium and Plant Laboratories (NHPL/KATH), Lalitpur.

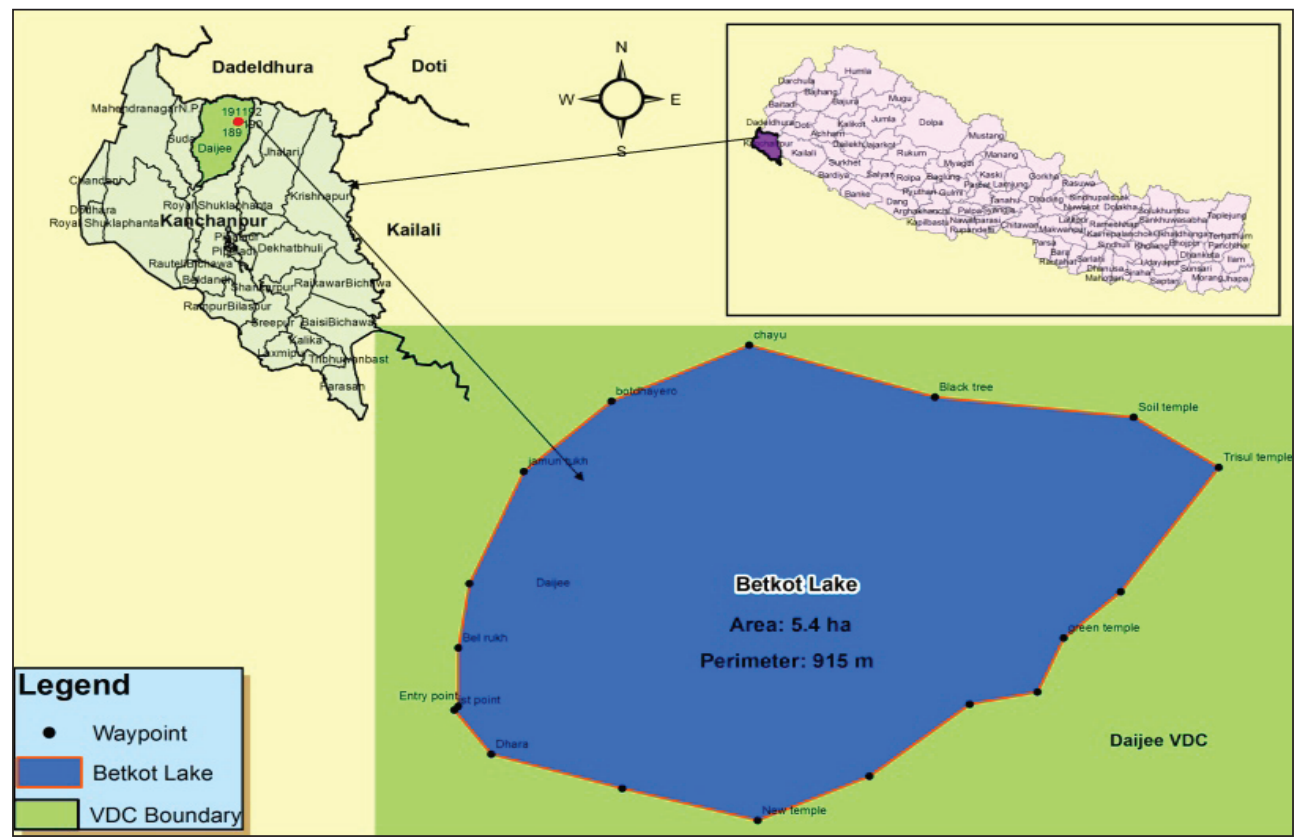

FIG. 1a. Map of study area. 


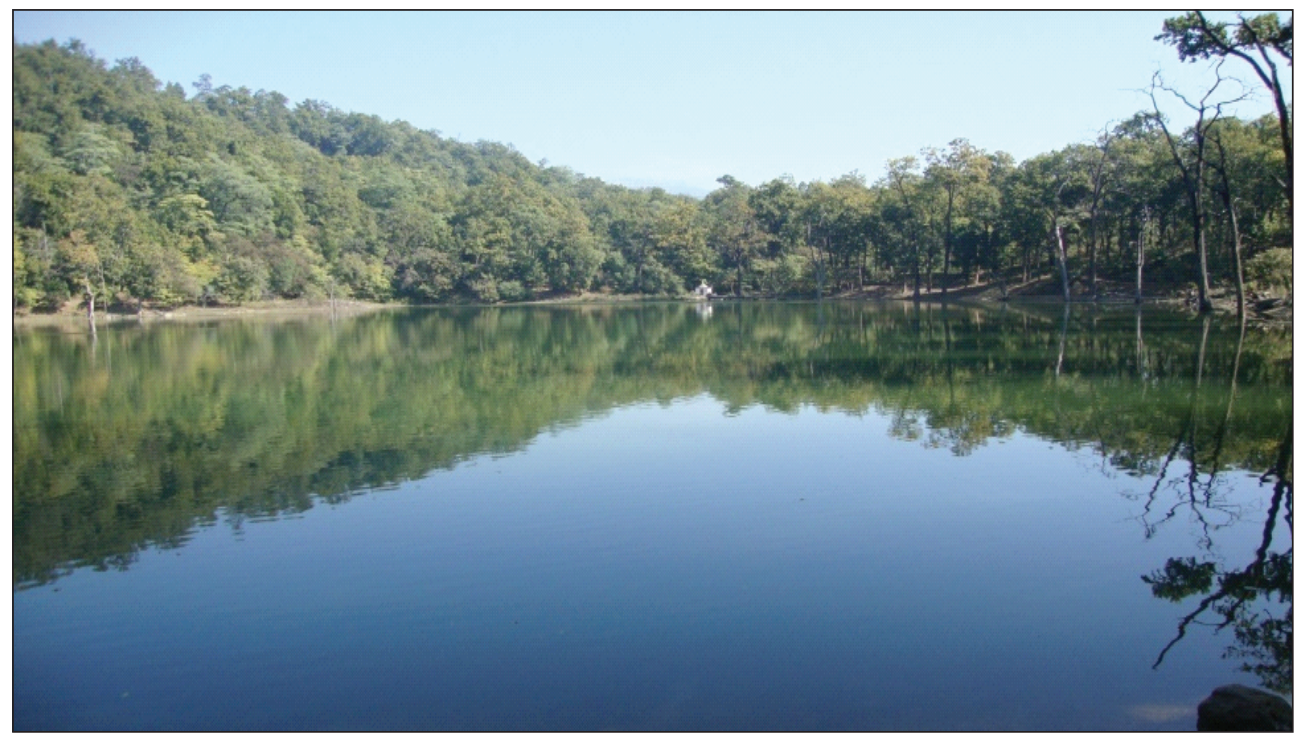

FIG. 1b. Betkotlake, Daijee-5, Kanchanpur, Nepal (Photo: BASNET, R;MAP: KARNA, Y K).

\section{RESULTS AND DISCUSSION}

A total of 63 plant species representing 40 families were recorded from the present study area (table 1). Of the total species, majority were angiosperms (60 species) and the rest were ferns ( 2 species) and mushroom ( 1 species). Asteraceae was the largest family with 6 species followed by Fabaceae (4 species), and Solanaceae, Malvaceae, Acanthaceae (3 species). The rest 35 families composed of 1-2 species. Among the total plants, Agele marmelos (Bel), Ficus religiosa (Pipal) and Sesamum indicum (Til) were the religious plants. These plants were occurred because of a great religious spot for the Hindus especially in 'Shiva Ratri' (Lord Shiva's Night). Likewise, present study also recorded some common medicinal plants such as Achyranthes aspera (Datiwan), Xeromphis spinosa (Mainphal), Syzygium cumini (Jamun), and Phyllanthus emblica (Amala). Quisqualis indica L. (Combretaceae) and Lygodium japonicum (Lygodiaceae) were the two climbers with aesthetic ornamental value. The uses of plants of Beeshazar Tal and Rampur Ghol was also reported as medicine, food, aesthetic, fodder, fuelwood, etc. (Dangol, 2000-2001; Dangolet al., 2014). The Ageratum conyzoides and Parthenium hysterophorus were two invasivespecies recorded in the study area. Different invasive species (such as Mikania micrantha, Ageratum houstonianum, Eichhornia crassipes) were reported from other wetlands of Nepal (Dangolet al., 2014).

Betkotlake shows typical tropical forest type with Shorea robusta, Haldina cordifolia, Mallotus philippensis, Syzygium cumini, and Bombax ceiba. This study added information on plant species which is helpful to understand the wetland flora. Further research is recommended to study the aquatic and semi-aquatic plants including algae of the area to understand the total plant life of the lake to generate the baseline information (local flora) for the 'Nepal Flora' publication. 
TABLE 1. Plant species from Betkotlake, far western Nepal.

\begin{tabular}{|c|c|c|c|c|c|}
\hline S.N. & Scientific name & Family & Local name & Life forms & $\begin{array}{c}\text { Coll. } \\
\text { nr. }\end{array}$ \\
\hline 1. & Achyranthes aspera L. & Amaranthaceae & Datiwan & Herb & $05 \mathrm{BT}$ \\
\hline 2. & Agele marmelos (L.) Corr-1 & Rutaceae & Bel & Tree & $09 \mathrm{BT}$ \\
\hline 3. & Ageratum conyzoides L. ${ }^{1}$ & Asteraceae & Gandhe & Herb & $022 \mathrm{BT}$ \\
\hline 4. & $\begin{array}{l}\text { Aleuritopteris bicolor (Roxb.) Fraser- } \\
\text { Jenk. }\end{array}$ & Pteridaceae & Fern & Herb & $031 \mathrm{BT}$ \\
\hline 5. & Amanita sp. & Amanitaceae & Chyau & Herb & 049 BT \\
\hline 6. & Arisaema intermedium BI. & Araceae & Sarpakomakai & Herb & $015 \mathrm{BT}$ \\
\hline 7. & Artemisia vulgaris $\mathrm{L}$. & Asteraceae & Pati & Herb & $031 \mathrm{BT}$ \\
\hline 8. & Biden spilosa L. & Asteraceae & Kurro & Herb & $032 \mathrm{BT}$ \\
\hline 9. & Bombax ceiba L. ${ }^{2}$ & Bombaceae & Simal & Tree & 044 BT \\
\hline 10. & Canscora decussate Schult. & Gentianaceae & Aakankuriphul & Herb & $037 \mathrm{BT}$ \\
\hline 11. & $\begin{array}{l}\text { Capillipedium assimile (Steud.) A. } \\
\text { Camus }\end{array}$ & Poaceae & Musa khari & Herb & $028 \mathrm{BT}$ \\
\hline 12. & Cassia fistula L. & Fabaceae & Rajbriskya & Tree & $035 \mathrm{BT}$ \\
\hline 13. & Cassia tora L. & Fabaceae & & Shrub & $033 \mathrm{BT}$ \\
\hline 14. & Centella asiatica (L.) Urb. & Apiaceae & Ghodtapre & Herb & 039 ВТ \\
\hline 15. & Cissamo pelospareira L. & Menispermaceae & Batulpate & $\begin{array}{l}\text { Climber } \\
\text { (Herb) }\end{array}$ & $038 \mathrm{BT}$ \\
\hline 16. & Clerodendrum indicum (L.) Kurtze & Verbenaceae & Bhargi & Shrub & $046 \mathrm{BT}$ \\
\hline 17. & Colebrooke aoppositifolia Sm. & Lamiaceae & Dhasure & Shrub & $036 \mathrm{BT}$ \\
\hline 18. & Colocasiae sculenta (L.) Schott. & Araceae & Jaluko & Herb & $033 \mathrm{BT}$ \\
\hline 19. & Crotalaria albidaHeyne ex. Roth. & Fabaceae & Putaliphul & Herb & $037 \mathrm{BT}$ \\
\hline 20. & Curculigo orchioides Gaertn. & Hypoxidaceae & Kalomusali & Herb & $055 \mathrm{BT}$ \\
\hline 21. & Cyperusco rymbosus Rottb. & Cyperaceae & Mothe & Herb & $062 \mathrm{BT}$ \\
\hline 22. & Dioscore abulbifera L. & Dioscoreaceae & Githetarul & Herb & 048 BT \\
\hline 23. & Diospyros smalabarica (Desr.) Kostel & Ebenaceae & Khallu/Tezu & Tree & $056 \mathrm{BT}$ \\
\hline 24. & $\begin{array}{l}\text { Diploknema butyraceae (Roxb.) H.J. } \\
\text { Lam. }^{3}\end{array}$ & Sapotaceae & Chiuri & Tree & 064 BT \\
\hline 25. & Ficus religiosa L. ${ }^{4}$ & Moraceae & Pipal & Tree & $039 \mathrm{BT}$ \\
\hline 26. & Flemingia paniculata Wall. ex. Benth. & Fabaceae & & Herb & 044 BT \\
\hline 27. & Gossypium arboreum L. & Malvaceae & Kapas & Tree & $07 \mathrm{BT}$ \\
\hline 28. & Grangea maderaspatana (L.) & Asteraceae & & Herb & $029 \mathrm{BT}$ \\
\hline
\end{tabular}




\begin{tabular}{|c|c|c|c|c|c|}
\hline 29. & $\begin{array}{l}\text { Haldina cordifolia (Willd.exRoxb.) } \\
\text { Ridsdale }\end{array}$ & Rubiaceae & Haldu/Karma & Tree & $08 \mathrm{BT}$ \\
\hline 30. & Hemidesmus indicus R.Br. & Asclepiadaceae & Anantmul & Herb & $040 \mathrm{BT}$ \\
\hline 31. & $\begin{array}{l}\text { Holarrhena pubescens (Buch.-Ham.) } \\
\text { Wall. ex G. Don }{ }^{5}\end{array}$ & Apocynaceae & Indrajau & Shrub & $031 \mathrm{BT}$ \\
\hline 32. & $\begin{array}{l}\text { Hygrophila auriaulata (Schumach) } \\
\text { Hein. }\end{array}$ & Acanthaceae & & Herb & $057 \mathrm{BT}$ \\
\hline 33. & Lagerstroemia parviflora Roxb. & Lythraceae & Bod Dhayro & Tree & $035 \mathrm{BT}$ \\
\hline 34. & $\begin{array}{l}\text { Lepidagathis incurva Buch.-Ham. ex } \\
\text { D. Don }\end{array}$ & Acanthaceae & & Herb & $03 \mathrm{BT}$ \\
\hline 35. & Lygodium japonicum (Thum.) Sw. & Lygodiaceae & Fern & $\begin{array}{l}\text { Climber } \\
\text { (Herb) }\end{array}$ & $017 \mathrm{BT}$ \\
\hline 36. & $\begin{array}{l}\text { Mallotus philippensis (Lam.) Muell.- } \\
\text { Arn. }\end{array}$ & Euphorbiaceae & Sindhure & Tree & $049 \mathrm{BT}$ \\
\hline 37. & Mangifera indica $\mathrm{L}$. & Anacardiaceae & Aanp & Tree & $050 \mathrm{BT}$ \\
\hline 38. & Murraya koenigii (L.) Spreng. & Rutaceae & Mithanim & Shrub & $044 \mathrm{BT}$ \\
\hline 39. & Nerium indicum $\mathrm{L}$. & Apocynaceae & Karvir & Shrub & $047 \mathrm{BT}$ \\
\hline 40. & Parthenium hysterophorus L. ${ }^{1}$ & Asteraceae & & Herb, & $011 \mathrm{BT}$ \\
\hline 41. & Persicaria barbata (L.) H.Hara & Polygonaceae & & Herb & $062 \mathrm{BT}$ \\
\hline 42. & Phyla nodiflora (L.) Greene & Verbenaceae & & Herb & $064 \mathrm{BT}$ \\
\hline 43. & Phyllanthus emblica L. ${ }^{5}$ & Euphorbiaceae & Amala & Tree & $040 \mathrm{BT}$ \\
\hline 44. & $\begin{array}{l}\text { Pogostemom benghalenis (Burm.f.) } \\
\text { Kuntze. }\end{array}$ & Lamiaceae & Rudilo & Herb & $057 \mathrm{BT}$ \\
\hline 45. & Psidium gujava L. & Myrtaceae & Amba & Tree & $034 \mathrm{BT}$ \\
\hline 46. & Quisqualis indica $\mathrm{L} .{ }^{6}$ & Combretaceae & & Climber & $025 \mathrm{BT}$ \\
\hline 47. & Rungia parviflora (Retz.) Nees & Acanthaceae & Ukuchejhar & Herb & $022 \mathrm{BT}$ \\
\hline 48. & Salix babylonica L. & Salicaceae & Bains & Tree & $053 \mathrm{BT}$ \\
\hline 49. & Schleichera oleosa (Lour.) Okm & Sapindaceae & Kusum & Tree & $039 \mathrm{BT}$ \\
\hline 50. & Semecarpus anacardium L.f. & Anacardiaceae & Bhalayo & Tree & $030 \mathrm{BT}$ \\
\hline 51. & Sesamum indicum L. ${ }^{4}$ & Pedaliaceae & Till & Herb & $04 \mathrm{BT}$ \\
\hline 52 & Shorea robusta Gaertn. ${ }^{7}$ & Dipterocarpaceae & Sal & Tree & $019 \mathrm{BT}$ \\
\hline 53. & Sida rhombifolia M. Wight \& Arn & Malvaceae & & Herb & $016 \mathrm{BT}$ \\
\hline 54. & Smilax zeylanicaL. & Liliaceae & Kukurdiano & $\begin{array}{l}\text { Climber } \\
\text { (Shrub) }\end{array}$ & $08 \mathrm{BT}$ \\
\hline
\end{tabular}


Basnet et al.: Wetland flora of Betkot lake, far western Nepal.

\begin{tabular}{|l|l|l|l|l|l|}
\hline 55. & Solanum americanum Mill. & Solanaceae & Kamai & Herb & 038 BT \\
\hline 56. & Solanum indicum L. & Solanaceae & & Shrub & 017 BT \\
\hline 57. & Solanum surattense Bu. & Solanaceae & Kanthakari & Shrub & 018 BT \\
\hline 58. & Spilanthes acmella (L.) L. & Asteraceae & & Herb & 040 BT \\
\hline 59. & Syzygium cumini (L.) Skeels & Myrtaceae & Jamun & Tree & 029 BT \\
\hline 60. & Urena lobata L. & Malvaceae & Bhalukuro & Herb & 028 BT \\
\hline 61. & Xeromphis spinosa (Thumb.) Keay & Rubiaceae & Main phal & Shrub & 019 BT \\
\hline 62. & Zingiber sp. & Zingiberaceae & & Herb & 049 BT \\
\hline 63. & Zizyphus mauritiana Lam. & Rhamnaceae & Bayer & Tree & 023 BT \\
\hline
\end{tabular}

${ }^{1}$ Invasive; , Fruit edible and religious value; ${ }^{2}$ Habitat for vulture; ${ }^{3}$ Fodder; ${ }^{4}$ Religious value; ${ }^{5}$ Medicinal value; ${ }^{6}$ Ornamental value; ${ }^{7}$ Timber value; and ${ }^{8}$ Fruit edible

These species represented 31 herbs, 16 shrubs, 15 trees and 3 climbers (fig. 1). Dominance of herbs in the wetland study areas in Nepal were also reported by other authors (Sah, 1997; Siwakoti, 2006; Dangol et al., 2014; Lamsal et al., 2014).

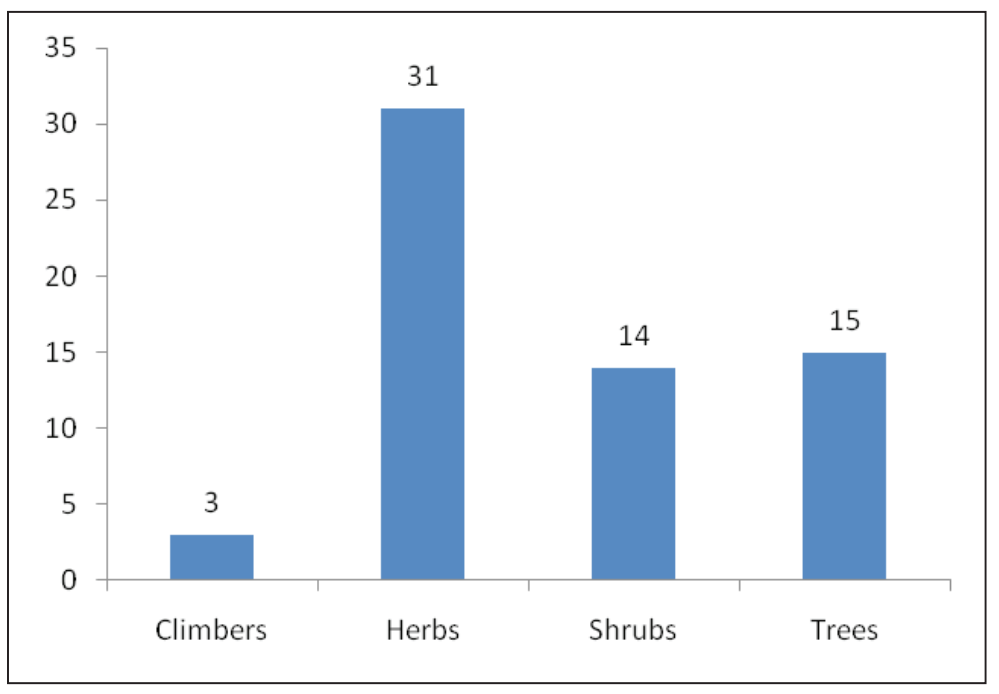

FIG. 1. Life form of plants of Betkotlake, far western Nepal.

\section{ACKNOWLEDGEMENTS}

We would like to thank to Dr. A.N. Das, former Director General of Department of Plant Resources (DPR), Kathmandufor his continuous encouragement of the study. Similarly, Dr. Nirmala Joshi (Scientific Officer), DPR, Mr. Tirtha Raj Pandey and Mr. Dhan Raj Kandel (Assistant Research Officers) of the National Herbarium and Plant Laboratories, Lalitpurareacknowledged for the plant identification.Prof. Dr. Dharma Raj Dangol of the Natural History Museum, Tribhuvan University, Kathmandu is acknowledged for providing 
valuable literatures and suggestions. Likewise, Mr. Yogendra Kumar Karna, Department of Forests, Kathmandu and Mr. Rabindra BC, former Assistant Botanist of the DPR are thanked for preparing the map and data respectively.

\section{REFERENCES}

BASNET, B; KHATRI, S; JOSHI, M D (2012) Documentation of wetland flora of hot springs in Bhurung-6, Myagdi, Nepal. Proceedings of International Wetland Symposium (IWS), Pokhara, Nepal held on November 7-9, 2012 organized by MOFSC/CSUWN, Nepal;pp 73-77.

BHANDARI, B (1998)An inventory of Nepal's Terai's wetlands. IUCN Nepal.

DANGOL, D R (2000-2001)Aquatic plant resources and their uses: observation from BeeshHazar Lake, Chitwan. Journal of Institute of Agriculture and Animal Science21-22: 119-133.

DANGOL, D R ; GAUTAM, B ; OLI, B B (2014) Wetland plants and their local uses: Observations from Rampur ghol, Chitwan, Nepal. Journal of Natural History Museum 28:142-159.

DPR (2001) Flowering plants of Nepal (Phanerogams), Department of Plant Resources (DPR), Kathmandu, Nepal.

DPR (2002)Pteridophytes of Nepal, Department of Plant Resources (DPR), Kathmandu, Nepal.

FRASER-JENKINS, C R; KANDEL, DR; PARIYAR, S (2015) Ferns and fern-allies of Nepal-1, National Herbarium and Plant Laboratories, Department of Plant Resources, Kathmandu, Nepal.

LAMSAL, P; PANT, K P; KUMAR, L;ATREYA, K (2014) Diversity, uses, and threats in the Ghodaghodi Lake complex, a Ramsar site in western lowland Nepal. ISRN Biodiversity.

MFSC (2002) Nepal biodiversity strategy. Ministry of Forests and Soil Conservation, Kathmandu, Nepal. PRESS, J R; SHRESTHA, K K; SUTTON, D A(2001)Annotated checklist of the flowering plants of Nepal. The Natural History Museum,London, UK.

SAH, J P (1997) KoshiTappu wetlands: Nepal's Ramsarsite.IUCN, Bangkok, Thailand.

SHRESTHA, K (1998) Dictionary of Nepalese plant names. Kathmandu, Nepal: Mandala Book Point, Kathmandu, Nepal.

SIWAKOTI, M (2006) An overview of floral diversity in wetlands of Terai region of Nepal. Our Nature 4: 83-90.

THAPA, N (2002) Pteridophytes of Nepal. Kathmandu, Nepal: Department of Plant Resources. Available at http://www.nepallake.gov.np/. 